\title{
Caracterización del componente ejecutivo en personal operativo vinculado al sector de hidrocarburos ${ }^{*}$
}

\author{
Jasmin Bonilla-Santos* \\ Alfredis Gonzalez-Hernandez $z^{* * *}$ \\ Sandra Beatriz Yepes-Artunduaga $a^{* * * *}$ \\ Leidy Johanna España-Parrasi ${ }^{* * * * *}$ \\ Examary Villarreal-Ruiz ${ }^{* * * * * *}$
}

Recibido: 30/01/2016 • Aceptado: 26/10/2016

DOI: 10.22395/rium.v16n31a11

\begin{abstract}
Resumen
La presente investigación se realizó en el marco del proyecto Caracterización del componente ejecutivo en personal operativo vinculado al sector de hidrocarburos del departamento del Huila en el año 2012. El objetivo fue describir el componente ejecutivo de 35 operarios pertenecientes a dicha población. Una de las dificultades es el manejo inadecuado de elementos de protección del personal operativo, lo que evidencia la necesidad de contar con instrumentos de valoración en procesos de selección de personal que permitan identificar las habilidades de anticipación, planeación y flexibilidad encargadas de evitar conductas de riesgo y promover comportamientos preventivos. Los resultados de la valoración neuropsicológica evidenciaron dificultades en los procesos de atención sostenida, sistema ejecutivo central, control inhibitorio y flexibilidad cognitiva. Se concluye que para el uso adecuado de los elementos de protección no es suficiente el conocimiento, sino el funcionamiento metacognitivo que regule el comportamiento de los sujetos para conservar la vida.
\end{abstract}

Palabras clave: elementos de protección; funciones ejecutivas; seguridad industrial; selección de personal.

Artículo derivado del proyecto de investigación Caracterización del componente ejecutivo en personal operativo vinculado al sector de hidrocarburos financiado por la Universidad Cooperativa de Colombia (Conadi), en el año 2013.

** Docente investigadora, coordinadora de investigación en Psicología, Universidad Cooperativa de Colombia. jasminbonillasantos@hotmail.com

*** Coordinador de Investigación Posgrados, Universidad Cooperativa de Colombia. alfredisg.h@hotmail.com

**** Docente investigadora, coordinadora Especialización Calidad y Auditoria y Salud, Universidad Cooperativa de Colombia.sandra.yepes@ucc.edu.co

****** Docente investigadora, Universidad Cooperativa de Colombia. leidy.espanap@ucc.edu.co

******* Docente investigadora, Universidad Cooperativa de Colombia. examary.villareal@campusucc.edu.co 


\title{
Characterization of the executive component in operational staff of the hydrocarbon sector
}

\begin{abstract}
This research was conducted with the project Characterization of the executive component in operational staff of Huila hydrocarbon sector, 2012. The objective was to describe the executive component of 35 operators belonging to such population. One of the difficulties is the improper management of protective elements by the operational staff; this implies a need for having valuation instruments in staff recruitment processes which allow identifying anticipation, planning, and flexibility skills needed to avoid risk conducts and promote preventive behavior. The results of the neuropsychological assessment showed difficulties for sustained attention processes, central executive system, inhibitory control, and cognitive flexibility. It was concluded that knowledge is not sufficient for the improper use of protective elements but the metacognitive functioning to regulate the behavior of the individuals in order to keep themselves safe.
\end{abstract}

Keywords: protective elements; executive functions; industrial security; staff recruitment.

\section{Caracterização do componente executivo em pessoal operativo vinculado ao setor de hidrocarbonetos}

\begin{abstract}
Resumo
Esta pesquisa foi realizada no âmbito do projeto Caracterização do componente executivo em pessoal operativo vinculado ao setor de hidrocarbonetos do estado de Huila em 2012. O objetivo foi descrever o componente executivo de 35 operários pertencentes a essa população. Uma das dificuldades foi a manipulação inadequada de elementos de proteção do pessoal operativo, o que evidencia a necessidade de contar com instrumentos de valoração em processos de seleção de pessoal que permitam identificar as habilidades de antecipação, planejamento e flexibilidade encarregadas de evitar comportamentos de risco e promover comportamentos preventivos. Os resultados da valoração neuropsicológica demonstraram dificuldades nos processos de atenção consistente, sistema executivo central, controle inibitório e flexibilidade cognitiva. Conclui-se que, para o uso adequado dos elementos de proteção, não é suficiente o conhecimento, mas sim o funcionamento metacognitivo que regulamente o comportamento dos sujeitos para conservar a vida.
\end{abstract}

Palavras-chave: elementos de proteção; funções executivas; segurança industrial; seleção de pessoal. 


\section{INTRODUCCIÓN}

Es accidente de trabajo todo suceso repentino que sobrevenga por causa o con ocasión del trabajo, y que produzca en el trabajador una lesión orgánica, una perturbación funcional o psiquiátrica, una invalidez o la muerte [1]. No obstante, en la actualidad mundial se presentan 337 millones de accidentes de trabajo [2], que según Cortés [3] de cada 100 accidentes, 85 se deben a los actos inseguros y solo uno a las condiciones inseguras; los restantes 14 accidentes ocurren por una combinación de ambos.

Para el caso de esta investigación, las principales dificultades que enfrentan a diario los supervisores de personal es la falta de concienciación del personal operativo respecto al uso de los elementos de protección, lo que incrementa la vulnerabilidad frente a los factores de riesgo. En España, García et al. [4] desarrollaron un modelo de predicción de la siniestralidad laboral basado en datos empíricos en un conjunto de variables susceptibles de intervención. El método empleado fue un diseño transversal y correlacional, en el que a partir de una muestra representativa de más de 500 trabajadores de diferentes sectores de actividad de la provincia de Valencia cuantificaron la importancia relativa de cada uno de los aspectos definidos como relevantes para predecir la ocurrencia de accidentes. En dicho estudio se concluye que los aspectos psicosociales son claves en la explicación de los accidentes laborales y abren vías para una intervención eficaz.

Una mirada desde la funcionalidad neuropsicológica explica las causas de accidentalidad en la toma de decisiones del uso inadecuado de los elementos de protección. Colombo et al. [5] refieren que el componente ejecutivo garantiza las habilidades para mantener una apropiada estrategia de resolución de problemas, las habilidades para llevar a cabo un plan estratégico de acción secuencial, las representaciones mentales de una tarea, la planeación, la auto-monitorización del comportamiento y las habilidades para usar las claves ambientales. A este respecto Lopera [6] expone que no es suficiente con tener una inteligencia normal y buenas habilidades cognitivas, sino que, además, es necesario tener un adecuado funcionamiento ejecutivo que controle y coordine la acción conjunta de todos los procesos cognitivos. Ello indica que el déficit ejecutivo traería consigo alteraciones en el comportamiento, las cuales se manifiestan en conductas impulsivas que se relacionan íntimamente con conductas de riesgo.

Los individuos actúan de forma impulsiva e ignoran información relevante para la actividad que deben realizar y obtienen como resultado graves consecuencias para la integridad física, además de traer graves implicaciones para la empresa y para el trabajador, quienes ven afectados su productividad, integridad y su economía, y hasta pueden tener implicaciones legales. La Organización Internacional del Trabajo señala como una de sus prioridades disminuir el alto índice de accidentalidad laboral ocurrido 
en todo el mundo [2]. De allí la importancia de concentrar esfuerzos para intervenir las posibles causas e implementar las medidas de control necesarias para mitigar los accidentes de trabajo.

Por esta razón, el presente estudio tuvo como objetivo principal caracterizar el componente ejecutivo en el personal operativo vinculado al sector de hidrocarburos del departamento del Huila en el año 2012, reportados con dificultades en la utilización de sus implementos de protección personal, con la finalidad de generar soluciones efectivas para prevenir accidentes de trabajo, e identificar los accidentes de causas básicas que son los que dan origen a las causas inmediatas; estas están integradas por factores personales (hábitos de trabajo incorrectos, uso incorrecto de equipos, herramientas e instalaciones; defectos físicos o mentales, deficiencias en la audición etc.) y por factores del trabajo (supervisión y liderazgo deficiente; políticas, procedimientos, guías o practicas inadecuadas; planeación y/o programación inadecuada del trabajo, etc.) $[7,8]$.

Teniendo en cuenta lo anterior podrían crearse nuevas políticas en seguridad industrial que contengan estrategias para monitorizar el desarrollo de las diferentes actividades y su procedimiento, observando la capacidad que presentan los operarios en la toma de decisiones, planeación y flexibilidad; además, la implementación de instrumentos que permitan evaluar el funcionamiento ejecutivo en los procesos de selección y lleven a la elección de un personal idóneo con capacidades de anticipación, de establecer metas, de diseñar planes y programas, con adecuada temporalidad y control de la conducta de acuerdo con los resultados de sus acciones, $[9,10]$.

\section{MATERIALES Y MÉTODOS}

Esta investigación se desarrolló desde un enfoque cuantitativo, con un diseño descriptivo de corte transversal. Se seleccionaron 35 sujetos del personal operativo vinculados a una empresa del sector de hidrocarburos del Huila, reportados por los supervisores como personal con dificultad para conservar los elementos de protección, a pesar de tener el conocimiento sobre la obligatoriedad y consecuencias de su uso inadecuado.

\section{Criterios de exclusión:}

- Limitación cognitiva

- Antecedente de trauma craneoencefálico o daño neurológico.

- Consumo de sustancias psicoactivas

- Vinculación menor a un año en el momento de la valoración.

Análisis estadístico: la muestra de estudio fue descrita en sus características demográficas: sexo, edad, escolaridad; se tuvo en cuenta la naturaleza de las variables con frecuencias, porcentajes, medidas de tendencia central y de variabilidad. 
Técnicas de recolección de instrumentos:

Instrumento de tamizaje diligenciado por los supervisores para la remisión del personal con las características solicitas.

Documento de recolección de información (historia clínica), que permite indagar por antecedentes de carácter clínico tales como: trauma craneoencefálico, Accidente cerebrovascular, accidentes laborales, o evento en el que haya estado cercano a la presentación de un accidente laboral.

\section{Instrumentos de valoración neuropsicológica: (componente ejecutivo)}

Test de asociación controlada de palabras (fluidez fonológica) FAS: se mide mediante el número de palabras producidas durante un minuto en una categoría específica solicitada, y es considerado una prueba de producción verbal controlada y programada, que es sensible a las alteraciones en el funcionamiento de las áreas prefrontales izquierdas; dentro del componente ejecutivo ha sido seleccionada como una prueba encaminada a valorar flexibilidad cognitiva [11]

Wisconsin Card Sorting Test: se utiliza este instrumento para evaluar la flexibilidad en el cambio de estrategias cognitivas y perseverancia cognitiva [12]. Esta prueba consiste en que se decide cuál es el principio (color, forma o número) que subyace a la agrupación de tarjetas, con la realimentación (correcta o incorrecta), que da el examinador a sus respuestas.

Trail Making Test (parte $A$ y $B$ ): es una prueba que sirve para evaluar la autorregulación, el control de la atención sostenida, y la capacidad de cambiar flexiblemente de una ejecución a otra. En general, el sujeto impulsivo comete numerosos errores, mientras que el intento demora más tiempo en la ejecución de la prueba [13].

Test de colores y palabras de Stroop: prueba de colores y palabras de Stroop. En neuropsicología clínica esta prueba permite medir la cualidad inhibitoria de las funciones. Este instrumento requiere que el paciente lea en una primera condición de administración (palabras) los nombres de tres colores (rojo, azul y verde). En una segunda condición de administración (colores) debe nombrar estímulos con cada uno de estos colores. Finalmente en la tercera condición de administración (palabras/colores o interferencia), el paciente debe decir el nombre del color con que está escrita la palabra, la que, a su vez, es el nombre de otro color [14].

La fiabilidad del Stroop se ha mostrado muy consistente en las distintas versiones existentes. En todos los casos, los investigadores han usado el método test-retest, con tiempos comprendidos entre un minuto y tres días entre las dos aplicaciones [15]. 


\section{RESULTADOS}

Como se observa en la tabla 1 , los elementos de protección con los cuales miembros del personal operativo de hidrocarburos del Huila son reportados por los supervisores como personas que presentan dificultad en su utilización son 11 y entre ellos los elementos más representativos son el capuchón con un 28,6\%; gafas de seguridad con un 17,1\%; tapaoídos de copa y de inserción con 14,3\% y 11,4\%, respectivamente.

Tabla 1: implementos de seguridad.

\begin{tabular}{l|l|c|c}
\hline \multicolumn{1}{c|}{ Implementos } & \multicolumn{1}{c|}{ Utilizacion } & Personas & Pocentaje \\
\hline \multirow{2}{*}{ Capuchón } & Tiene pero no usa & 10 & 28,6 \\
\hline \multirow{2}{*}{ Gafas de seguridad } & Tiene pero no usa & 6 & 17,1 \\
\cline { 2 - 4 } & Usa pero no es adecuado & 1 & 2,9 \\
\cline { 2 - 4 } & Usa incorrectamente & 1 & 2,9 \\
\hline Careta de soldadura & Tiene pero no usa & 1 & 2,9 \\
\hline Tapaoídos de copa & Tiene pero no usa & 5 & 14,3 \\
\hline Tapaoídos de inserción & Tiene pero no usa & 2 & 11,4 \\
\hline \multirow{2}{*}{ Mascarilla de polvo } & Tiene pero no usa & 1 & 2,7 \\
\cline { 2 - 4 } & Mal estado & 1 & 2,9 \\
\hline Mascarilla para humos & Tiene pero no lo usa & 2 & 5,7 \\
\hline Mascarilla con cartucho & Tiene pero no lo usa & 1 & 2,9 \\
\hline Guantes de vaqueta & Tiene pero no lo usa & 1 & 2,9 \\
\hline Guantes de nitrilo & Tiene pero no lo usa & 1 & 2,9 \\
\hline Mangas de vaqueta & Tiene pero no lo usa & & 2,9 \\
\hline
\end{tabular}

Fuente: elaboración propia

\section{Características de la población}

La tabla 2, permite señalar que la edad y la escolaridad no se encuentran afectadas por datos extremos y que existe homogeneidad en cuanto a estas variables.

Tabla 2: edad y escolaridad de los participantes.

\begin{tabular}{l|c|c|c|cc}
\hline & $N$ & Media & Mediana & Desviación típica & Valor P \\
\hline Edad & 35 & 42 & 43 & 12,42 & 0,29 \\
\hline Escolaridad & 35 & 10,8 & 12 & 3 & 0,63 \\
\hline
\end{tabular}




\section{Valoración de los procesos ejecutivos}

En la tabla 3 se comparó la puntuación obtenida por la muestra, respecto a la señalada en la baremación colombiana para este tipo de prueba; se observó que existe diferencia significativa y que estas son a favor de la media poblacional, lo que indica dificultades en el proceso de atención sostenida, esto es en la capacidad de mantenernos alerta en una sola tarea por un tiempo prolongado.

Tabla 3: valoración atención sostenida

\begin{tabular}{l|c|c|c|cc|c}
\hline \multicolumn{1}{c|}{ Pruebas } & $N$ & Media & $\bar{X}$ poblacional & Des. Tip & $\begin{array}{c}\text { Des.Tip } \\
\text { Poblacional }\end{array}$ & Valor P \\
\hline TMT A (Aciertos) & 35 & 23,14 & 24 & 1,89 & & 0,00 \\
\hline TMT A (Tiempo) & 35 & 80,77 & 45,18 & 25,14 & 24,06 & 0,00 \\
\hline Dígitos en progresión & 35 & 5,43 & 5,1 & 0,91 & & 0,006 \\
\hline
\end{tabular}

Fuente: elaboración propia

En la evaluación de memoria de trabajo (tabla 4), se presentan diferencia significativa a favor del grupo de estudio, indicando que no presentan dificultades en memoria de trabajo.

Tabla 4: memoria de trabajo

\begin{tabular}{|l|c|c|c|c|c|}
\hline \multicolumn{1}{|c|}{ Pruebas } & $N$ & Media & $\bar{X}$ Poblacional & Des. tip & Valor P \\
\hline Dígitos regresión & 35 & 3,71 & 3,2 & 1,22 & 0,025 \\
\hline Dígitos en progresión & 35 & 5,43 & 5,1 & 0,91 & 0,006 \\
\hline
\end{tabular}

Fuente: elaboración propia

Al comparar las puntuaciones obtenidas por la muestra con lo referenciado para la población colombiana, en la tabla 5 se presenta diferencias significativas que no favorecen al grupo en estudio, indicando dificultad para la realización de dos tareas simultáneas o concurrentes, es decir, presentan dificultad para alternar y dividir su foco atencional.

Tabla 5: fluidez verbal.

\begin{tabular}{c|c|c|c|cc|c}
\hline Pruebas & $\mathrm{N}$ & Media & $\bar{X}$ Poblacional & Des. Tip & $\begin{array}{c}\text { Des. Tip } \\
\text { Poblacional }\end{array}$ & Valor P \\
\hline FAS & 35 & 27,14 & 33,4 & 9,36 & 10,13 & 0,00 \\
\hline
\end{tabular}

Fuente: elaboración propia 
Al comparar la puntuación obtenida por la muestra con lo referenciado para la población colombiana, en la tabla 6 se presenta que existe diferencia significativa, en el control inhibitorio.

Tabla 6: control inhibitorio.

\begin{tabular}{|l|c|c|c|c|c|c|}
\hline \multicolumn{1}{|c|}{ Pruebas } & $N$ & Media & $\bar{X}$ Poblacional & Des. Tip & $\begin{array}{c}\text { Des. Tip } \\
\text { Poblacional }\end{array}$ & Valor P \\
\hline Stroop P & 35 & 92,89 & 85,82 & 14,97 & 18,16 & 0,001 \\
\hline Stroop C & 35 & 64,11 & 65,18 & 14,17 & 13,47 & 0,482 \\
\hline Stroop P vs C & 35 & 35,63 & 39,79 & 8,18 & 11,29 & 0,003 \\
\hline
\end{tabular}

Fuente: elaboración propia

Al comparar la puntuación obtenida por la muestra respecto a la señalada en la baremación colombiana, en la tabla 7 se observaron dificultades en el proceso de flexibilidad cognitiva.

Tabla 7: flexibilidad cognitiva.

\begin{tabular}{|l|c|c|c|c|c|c|}
\hline \multicolumn{1}{|c|}{ Pruebas } & $N$ & Media & $\bar{X}$ Poblacional & Des. Tip & $\begin{array}{c}\text { Desv. Tip } \\
\text { Poblacional }\end{array}$ & Valor P \\
\hline Categorías & 35 & 3,31 & 5,03 & 2,33 & 1,55 & 0,00 \\
\hline Total de errores & 35 & 49,45 & 31,36 & 20,42 & 20,85 & 0,00 \\
\hline Errores perseveración & 35 & 22,17 & 20,14 & 10,99 & 18,51 & 0,338 \\
\hline TMT B (Aciertos) & 35 & 20,45 & 24 & 5,14 & & 0,00 \\
\hline TMT B (Tiempo) & 35 & 139,11 & 101,21 & 67,06 & 59 & 0,00 \\
\hline
\end{tabular}

Fuente: elaboración propia

\section{DISCUSIÓN}

Los resultados obtenidos en la presente investigación permitieron analizar los factores neurocognitivos de procesamiento superior y factores cognitivos de conocimiento que están implicados en los accidentes de trabajo por el uso inadecuado de los elementos personales de protección. Se pudo determinar que en la valoración de las variables cognitivas no hay diferencia respecto a la población normalizada, pero en cuanto a las variables de tipo metacognitivo esta población se encuentra por debajo de lo normalizado. Debido a que en los accidentes de trabajo es fundamental el reconocimiento y control de las causas básicas que dan origen a las causas inmediatas, estas últimas, integradas por factores personales (hábitos de trabajo incorrectos, uso incorrecto de equipos, herramientas e instalaciones; defectos físicos o mentales, deficiencias en la audición etc.) y por factores del trabajo (supervisión y liderazgo deficiente; políticas, 
procedimientos, guías o practicas inadecuadas; planeación y/o programación inadecuada del trabajo, etc.) [6], es necesario que las empresas realicen modificación en sus procesos de entrenamiento y capacitación que lleven a la disminución de los riesgos laborales y a la prevención de sus accidentes.

Haciendo un análisis detallado del proceso de valoración de los 35 operarios identificados por los supervisores como personal con dificultad para la utilización de los elementos personales de protección, se identificó que el $28,6 \%$ no utilizaban el capuchón; gafas de seguridad con un 17,1\%; tapaoídos de copa y de inserción con 14,3\% y 11,4\% respectivamente; estos datos estadísticos fueron similares a los aportados por otras investigaciones como la desarrollada por Salazar et al. [16] relacionada con la seguridad, salud y percepción de factores personales y organizacionales en la industria manufacturera de España, en el cual fue aplicado un cuestionario a 24 industrias de pequeña, mediana y grande empresa, que dio como resultados que solo el $25 \%$ de la población de mediana y pequeña empresa utilizaba correctamente los equipos de protección personal; otro estudio que reitera la falta del uso de los implementos de protección, con conocimiento previo de su importancia, es el realizado por Arias [17], que concluye que el $37 \%$ del personal que trabaja en construcción no utiliza los implementos por la incomodidad que estos causan y que el $29 \%$ no los utiliza debido a que dificultan la realización de ciertas actividades; los tapaoídos y las gafas son los menos utilizados.

Respecto a la misma población, Villalobos y Zúñiga [18], al realizar un estudio a 10 trabajadores de una empresa productora de papel, indicaron que, aunque en la empresa se siguieran los lineamientos del Sistema General de Riesgos Profesionales en Colombia y los trabajadores accidentados tuvieran el conocimiento completo de los riesgos y el deseo de protegerse, no lograron articular estos aspectos para dirigir sus acciones en el momento de la situación de riesgo, es decir, no pudieron anticipar las consecuencias que traía el no uso adecuado de los elementos personales de protección, lo que podría significar una lesión momentánea o de por vida; esto posiblemente relacionado con la dificultad de planear su conducta al pensar solo en cumplir con su tarea o actividad sin importar o dedicar tiempo al cómo lo va hacer, con qué lo va a realizar y qué cosas adicionales necesita.

Las investigaciones mencionadas corroboran los hallazgos y los objetivos que se plantearon en este estudio, porque, al igual que los trabajadores de estas empresas, los operarios de esta investigación no presentan dificultades en el nivel de la cognición como conocimiento o información, ni en la memorización de los datos (memoria de trabajo: dígitos en progresión y regresión); el problema se centra en los componentes metacognitivos que requieren de un procesamiento superior y complejo que, a su vez, 
involucra otras áreas cerebrales en especial las frontales que son las encargadas del control de la conducta humana, de actuar de manera correcta $[19,20]$.

Respecto al funcionamiento ejecutivo que presentan los seres humanos en las labores de la vida diaria, este sistema garantiza las habilidades para mantener una apropiada estrategia de resolución de problemas para el logro de una meta, la capacidad de llevar a cabo un plan estratégico de acción secuencial, las representaciones mentales de una tarea, la auto-monitorización del comportamiento y las habilidades para usar las claves ambientales. Es decir, continuamente se realimentan de las situaciones del medio, evalúan los riesgos y exploran las consecuencias futuras de sus comportamientos.

En los resultados obtenidos de la valoración del sistema ejecutivo central realizado con el test de fluidez verbal, los operarios presentaron fallas respecto a la población normalizada, posiblemente por el requerimiento de la prueba en planear la estrategia adecuada en tiempo, comodidad y control mental; con respecto a la flexibilidad cognitiva, los operarios presentan dificultad para la ejecución de dos tareas de manera simultánea o alternar su foco atencional pasando de una tarea a otra; de igual forma, el proceso de control inhibitorio y de anticipación evidencia que los operarios presentan dificultad para hacer control de las respuestas automáticas o predominantes, y para anticipar situaciones novedosas, con un esquema de acción y pensamiento fijo.

Estos resultados determinan que no es suficiente el entrenamiento a los operarios y el conocimiento de los diferentes tipos de riesgo que enfrentan en la empresa, sino que también existen elementos estructurales que producen un déficit ejecutivo que lleva a cometer el acto irresponsable de no protección [21]. En este sentido se recomienda incluir en los procedimientos organizacionales para el proceso de selección de personal la evaluación del componente metacognitivo, aun para cargos de tipo operativo con el fin de minimizar el riesgo de accidentalidad.

\section{CONCLUSIONES}

Con los hallazgos de la investigación se sugiere la implementación de procedimientos organizacionales en la selección del personal que incluyan la valoración de los procesos ejecutivos. Respecto a la inducción y reinducción es importante garantizar no solo el entrenamiento y conocimiento de los procedimientos institucionales, sino, además, la implementación de programas continuos de simulación que desarrollen habilidades en la auto-monitorización de la conducta y la realimentación de las tareas, y finalmente en la toma de decisiones con la evaluación de las consecuencias de la acción, con el fin de generar una cultura de la prevención, orientada a la disminución de los índices de accidentalidad y enfermedad en el entorno laboral y la mortalidad de los trabajadores. 


\section{AGRADECIMIENTOS}

Los autores expresan sus agradecimientos al Comité Nacional para el Desarrollo de la Investigación (CONADI) de la Universidad Cooperativa de Colombia por la financiación del estudio.

\section{REFERENCIAS}

[1] Ley 1562 de 2012, "Por la cual se modifica el Sistema de Riesgos Laborales y se dictan otras disposiciones en materia de Salud Ocupacional”. [En línea]. Disponible http://www.alcaldiabogota.gov.co/sisjur/normas/Norma1.jsp?i=48365, 2012

[2] Organización Internacional del Trabajo, "La prevención: Una estrategia global”. [En línea]. Disponible: https://www.ilo.org/legacy/english/protection/safework/worldday/products05/ report05_sp.pdf, 2005

[3] J. Cortés. Seguridad e higiene del trabajo: técnicas de prevención de riesgos laborales. México: Alfaomega, 2002.

[4], M. García-Layunta et al. Factores psicosociales influyentes en la ocurrencia de accidentes laborales" Prev Riesgos Labor; Vol. 5, n. ${ }^{\circ}$ 1, pp. 4-10, 2002

[5] E. M. Colombo et al. "Función ejecutiva y conductas impulsivas". Presentado en Cuarto Congreso Virtual Interpsiquis, 2003

[6] F. Lopera "Funciones ejecutivas: aspectos clínicos" Revista Neuropsicología, Neuropsiquiatría y Neurociencias, Vol.8, n. ${ }^{\circ}$, pp. 59-76, 2008

[7] R. Chinchilla. Salud y seguridad en el trabajo. [En línea]. Disponible: http://books.google. com.co/books?id=Y35TDM74KmUC\&printsec=frontcover\&hl=es\&source=gbs_ge_summa ry_r\&cad $=0 \# \mathrm{v}=$ onepage $\& \mathrm{q} \& \mathrm{f}=$ false, 2002

[8] Instituto Nacional de seguridad e higiene en el trabajo. "Causas de accidente: clasificación y codificación. Notas técnicas de prevención. [En línea]. Disponible: http://www.insht.es/ InshtWeb/Contenidos/Documentacion/NTP/NTP/Ficheros/891a925/924w.pdf, 2002

[9] E. Fernández et al. "Evaluación de factores de riesgo biopsicosociales asociados a la accidentabilidad laboral en empresas de producción". [Tesis]. Cali: Pontificia Universidad Javeriana, Facultad de Humanidades y Ciencias Sociales, 2006.

[10] N. Trujillo y D. Pineda. "Función ejecutiva en la investigación de los trastornos del comportamiento del niño y del adolescente. Colombia” Revista Neuropsicología, Neuropsiquiatría y Neurociencias. Vol. 18, n. ${ }^{\circ}$ 1, pp. 77-94, 2008

[11] J. A. Pino y R. Allegri. Interpretación neuropsicológica del test de asociación controlada de palabras. monografías de evaluación neuropsicológica. Florida, Estados Unidos: Interamerican Academy of Applied Cognitive Neurosciece, 2007.

[12] R. Heaton et al. Test de clasificación de tarjetas de Wisconsin. (WCST). (2 ed.). Manual: TEA Ediciones, 1948 
[13] Reitan. Trial Making Test. Parte A y parte B, 1958.

[14] A. Ardila y M. Daponte. Neuropsicología Clinica. Bogotá: Manual Moderno, 2007.

[15] C. Golden. STROOP: Test de Colores y Palabras [manual prueba psicológica]. Madrid: TEA Ediciones, 2001

[16] J. Salazar et al. "Seguridad, salud y percepción de factores personales y organizacionales en la industria manufacturera", Cienc Trab., [En línea], Vol. 12, n. ${ }^{\circ}$ 38, pp. 397-402. Disponible: http://www.fisoweb.org/imagenes/publicaciones/archivos/3115.pdf, 2010

[17] W. Arias. "Uso y desuso de los equipos de protección personal en trabajadores de construcción”. Cienc Trab., [En línea], Vol. 13, n. ${ }^{\circ}$ 40, pp. 119- 124. [En línea]. Disponible: http://www. estrucplan.com.ar/articulos/verarticulo.asp?IDArticulo=2778, 2011

[18] M. Villalobos y W. Zúñiga. "Perspectiva clínica de la consciencia del riesgo en la accidentalidad laboral: un estudio cualitativo". Pensamiento psicológico, [En línea], Vol. 9, n. ${ }^{\circ}$ 16, pp. 55-68. [En línea]. Disponible: http://www.redalyc.org/articulo.oa?id=80118612004, 2011

[19] J. Flores y F. Ostrosky. "Neuropsicología de Lóbulos Frontales, Funciones Ejecutivas y Conducta Humana”. Revista Neuropsicología, Neuropsiquiatría y Neurociencias, [En línea], Vol. 8, n. ${ }^{\circ}$ 1, pp. 7-58. Disponible: neurociencias.udea.edu.co/revista/PDF/REVNEURO_vol8_num1_7. pdf, 2008

[20] M. Ardila et al. Efectos del estrés sobre las funciones cognoscitivas en operarios del sector mecánico automotriz de vehículos pesados en la ciudad de Bogotá. Pontificia Universidad Javeriana (tesis). [En línea]. Disponible: http://repository.javeriana.edu.co/bitstream/10554/15546/1/ ArdilaNinoMonicaLiliana2014.pdf, 2014

[21] Mujica, A. "Nuevo objetivo en la mira de la neuropsiquiatría: el síndrome disejecutivo y disejecutividad. Hallazgos clínicos neurológicos y psiquiátricos, Chile”. Revista Chilena de Neuropsicología, [En línea], vol. 6, n. 1, pp. 7-11. Disponible: http://www.redalyc.org/articulo. oa? id=179322533002, 2011 